\title{
In vitro effects of Indian ayurvedic medicinal preparation against UV B induced cytotoxicity
}

\section{ANUPSingh Thakur and SonU AmbWani}

\begin{abstract}
The object of the present study was to investigate the effect(s) of UV-B irradiation on chicken splenocyte culture and the ameliorating effect of Indian Ayurvedic medicinal preparation. The medicinal preparation i.e. aqueous extracts from Andrographis pnaiculata (Acanthaceae) and hydromethanolic extract of Acacia catechu (Fabaceae) namely ANDRO and ACQ, were used against UV B rays induced cytotoxicity. Isolated lymphocytes were subjected to UV-B irradiation and in combination of medicinal preparations in vitro for $0,250,500,1250,2500$ and $3750 \mathrm{Joules} / \mathrm{m}^{2}$ which correspond to the irradiation time of $0,1,2,5,10$ and 15 minutes. 3-[4,5-dimethylthiozol-2yl]-2,5-diphenyltetrazolium bromide [MTT] assay performed for viability of lymphocytes in both treatments. Result show that the significant reduction in cell viability with increase in exposure of UV B rays. While the treatment of medicinal plants i.e. the dose of $0.3 \mathrm{mg} / \mathrm{ml}$ of both ANDRO and ACQ significantly reduces the per cent reduction in cell viability due to exposure of UV B rays.
\end{abstract}

Key words : Ultraviolet B irradiation, Viability

How to cite this paper : Thakur, Anupsingh and Ambwani, Sonu (2015). In vitro effects of Indian ayurvedic medicinal preparation against UV B induced cytotoxicity. Ann. Pharm. \& Pharm. Sci., 6 (1\&2) : 1-7.

Article chronicle : Received : 10.02.2015; Revised : 15.08.2015; Accepted : 10.09.2015 